\title{
Two-dimensional QCD in the Wu-Mandelstam-Leibbrandt prescription
}

\author{
Matthias Staudacher* \\ CERN, Theory Division, CH-1211 Geneva 23, Switzerland
}

Werner Krauth

CNRS-Laboratoire de Physique Statistique, Ecole Normale Supérieure, 24, rue Lhomond, 75231 Paris Cedex 05, France

(Received 15 September 1997; published 21 January 1998)

\begin{abstract}
We find the exact nonperturbative expression for a simple Wilson loop of arbitrary shape for $\mathrm{U}(N)$ and $\mathrm{SU}(N)$ Euclidean or Minkowskian two-dimensional Yang-Mills $\left(\mathrm{YM}_{2}\right)$ theory regulated by the $\mathrm{Wu}$ Mandelstam-Leibbrandt gauge prescription. The result differs from the standard pure exponential area law of $\mathrm{YM}_{2}$ theory, but still exhibits confinement as well as invariance under area-preserving diffeomorphisms and generalized axial gauge transformations. We show that the large $N$ limit is not a good approximation to the model at finite $N$ and conclude that Wu's $N=\infty$ Bethe-Salpeter equation for two-dimensional QCD should have no bound state solutions. The main significance of our results derives from the importance of the Wu-Mandelstam-Leibbrandt prescription in higher-dimensional perturbative gauge theory. [S0556-2821(98)00206-9]

PACS number(s): 11.10.Kk, 11.15.Pg, 12.38.Cy
\end{abstract}

Two-dimensional QCD $\left(\mathrm{QCD}_{2}\right)$, the dynamical theory of quarks and gluons in two dimensions, has been a fascinating testing-ground for some of the important concepts of high energy physics. A profound analysis of the theory was given by 't Hooft [1] in the limit $N \rightarrow \infty$ corresponding to an infinite number of colors. 't Hooft's study has culminated in the explicit demonstration of quark confinement and in the numerical determination of the bound state meson spectrum.

Technically, 't Hooft has worked in a generalized axial gauge, which means that the gauge potential is set to zero along a fixed direction. This direction is most conveniently chosen as lightlike. Within this so-called light-cone gauge $\left(A_{-}=0\right)$, one of the two quark spinor components decouples. 't Hooft has treated the remaining infrared singularity of the gauge field propagator in a way equivalent (cf. [2]) to a principal part prescription:

$$
\frac{1}{k_{-}^{2}} \rightarrow \frac{\mathcal{P}}{k_{-}^{2}}=\frac{1}{2}\left[\frac{1}{\left(k_{-}+i \epsilon\right)^{2}}+\frac{1}{\left(k_{-}-i \epsilon\right)^{2}}\right]
$$

The highly consistent 't Hooft theory has been extended to many studies. In particular, the invariance of the meson spectrum with respect to a different choice of gauge, the space-like axial gauge, has been checked explicitly by a combination of analytical and numerical calculations [3]. In this more complicated gauge one has to deal with both quark spinor components, and the integral equation for the meson bound states becomes two-dimensional. Nevertheless, an identical bound state meson spectrum was obtained [3].

\footnotetext{
*Present address: Max-Planck Institut für Gravitationsforschung, Schlaatzweg 1, D-14473 Potsdam, Germany. Email address: matthias@nxth04.cern.ch

${ }^{\dagger}$ Email address: krauth@physique.ens.fr
}

$\mathrm{QCD}_{2}$ would appear to be completely satisfactory were it not for two potential problems, which turn out to be closely related. The first is that, using the principal part prescription (1), Wick rotation to Euclidean space is impossible [4]. This was remarked early on by $\mathrm{Wu}$ [5], who suggested to formulate $\mathrm{QCD}_{2}$ in Euclidean space. He considered the Wickrotated kernel $\left(k_{-}=k_{1}-k_{0} \rightarrow k_{1}+i k_{2}\right)$

$$
\frac{1}{k_{-}^{2}} \rightarrow \frac{1}{\left(k_{1}+i k_{2}\right)^{2}}
$$

and proposed to use symmetric integration. This means that the integrals with the kernel Eq. (2) should exclude a small spherical hole of radius $\epsilon$ around the origin of the $\left(k_{1}, k_{2}\right)$ plane.

Secondly, the principal part prescription for generalized axial gauges appears to be irreparably inconsistent above two dimensions since manifest perturbative renormalizability is lost. For light-cone gauges above two dimension, the regularization suggested by Mandelstam [6] and by Leibbrandt [7] appears to solve this problem (cf. also [8]). In two dimensions, this prescription gives

$$
\frac{1}{k_{-}^{2}} \rightarrow \frac{1}{\left(k_{-}-i \epsilon \operatorname{sgn} k_{+}\right)^{2}}=\frac{\mathcal{P}}{k_{-}^{2}}-i \pi \delta^{\prime}\left(k_{-}\right) \operatorname{sgn} k_{+} \cdot
$$

Bassetto et al. [9] noted that the $\mathrm{Wu}$ kernel (2) and the Mandelstam-Leibbrandt kernel (3) are related to each other by a Wick rotation.

$\mathrm{Wu}$ [5] derived an effective integral equation for the bound state mesons in $\mathrm{QCD}_{2}$ at $N=\infty$ with the regularization of Eqs. (2), (3). Physical observables should not depend on the regularization scheme, and it was widely suspected that the Wu-Mandelstam-Leibbrandt (WML) kernels should lead 
to the same results as the 't Hooft prescription. Past efforts to solve the $\mathrm{Wu}$ equation have however failed. For some early investigations see [10].

The apparent impossibility to resolve the meson bound state equation in the $\mathrm{Wu}$ formalism is not a true impediment to study $\mathrm{QCD}_{2}$ in the WML prescription. Complementary insight can be obtained by probing the Yang-Mills field by static flux lines instead of dynamical quarks: the Wilson loops. This has already been the basic motivation of work by Bassetto et al. [11].

The present paper consists in a study of Wilson loops for $\mathrm{QCD}_{2}$. We first investigate perturbatively various contours in Euclidean and Minkowski space, up to $\mathcal{O}\left(g^{6}\right)$, using numerical and analytic calculations. We then derive the exact nonperturbative result. Our study confirms explicitly the finding [11] that the WML regularization gives a result different from 't Hooft's. However, contrary to what was claimed before in the literature [11], the result satisfies the same consistency conditions as 't Hooft's Wilson loop: (i) it does not depend on the shape of the closed contour, but only on its area, (ii) the leading behavior at finite $N$ is an area law behavior, indicating confinement, and (iii) the result is gauge invariant in a (restricted) way which will be detailed later on. In our opinion, these tests are evidence that the WML prescription gives rise to a self-consistent two-dimensional gauge theory. This theory is, however, not standard twodimensional QCD as described by the 't Hooft prescription.

Before endeavoring on the detailed calculations in the WML regularization, we briefly present the precise definitions and review the main results of the analogous calculation using 't Hooft's prescription. Wilson loops are defined as

$$
\mathcal{W}_{\mathcal{C}}=\left\langle\frac{1}{N} P \operatorname{Trexp}\left(i g \oint_{\mathcal{C}} d \vec{x} \cdot \vec{A}\right)\right\rangle
$$

Here $P$ denotes path ordering of the gauge field $\vec{A}$ along the contour $\mathcal{C}$. In two Euclidean dimensions the standard, exact result can be obtained in a gauge invariant way for any number of colors and by a variety of methods; e.g., using a manifest gauge invariant formulation like lattice gauge theory. For the case of a simple (i.e., not self-intersecting) $\mathrm{U}(N)$ or $\mathrm{SU}(N)$ Wilson loop $\mathcal{W}_{\mathcal{C}}$ along a contour $\mathcal{C}$ one obtains

$\mathcal{W}_{\mathcal{C}}^{\mathrm{U}(N)}=e^{-(1 / 2) N g^{2} \mathcal{A}_{\mathcal{C}}}, \quad \mathcal{W}_{\mathcal{C}}^{\mathrm{SU}(N)}=e^{-(1 / 2)(N-1 / N) g^{2} \mathcal{A}_{\mathcal{C}}}$,

where $\mathcal{A}_{\mathcal{C}}$ is the area enclosed by $\mathcal{C}$. Apart from the trivial factor $N$, the $\mathrm{U}(N)$ result does not distinguish between the Abelian and the non-Abelian case. Furthermore, the form of Eq. (5) agrees with the idea of a linear confining potential between sources. $\mathcal{W}_{\mathcal{C}}$ depends on the contour $\mathcal{C}$ solely through the dimensionless combination $g^{2} \mathcal{A}_{\mathcal{C}}$. The general reasons for this fact were first emphasized in [12]: $\mathrm{YM}_{2}$ is invariant under area preserving diffeomorphisms [13]. Even in Minkowski space, the 't Hooft prescription (1) (or any other generalized axial gauge with principal part prescription) reproduces the exponential law (5) with $\mathcal{A}_{\mathcal{C}} \rightarrow i \widetilde{\mathcal{A}}_{\mathcal{C}}$ where $\widetilde{\mathcal{A}}_{\mathcal{C}}$ is the Minkowski "area", enclosed by the contour.
Let us first work in Euclidean space and use the prescription (2). The path-ordered exponential in Eq. (4) is defined as

$$
\begin{aligned}
\mathcal{W}_{\mathcal{C}}= & \frac{1}{N_{n=0}} \sum_{0}^{\infty}\left(-g^{2}\right)^{n} \int_{0}^{1} d s_{1} \dot{x}_{-}\left(s_{1}\right) \cdots \\
& \times \int_{0}^{s_{2 n-1}} d s_{2 n} \dot{x}_{-}\left(s_{2 n}\right) \operatorname{Tr}\left\langle A_{+}\left(\vec{x}\left(s_{1}\right)\right) \cdots A_{+}\left(\vec{x}\left(s_{2 n}\right)\right)\right\rangle,
\end{aligned}
$$

where $\vec{x}(s), \quad s \in[0,1]$ parametrizes the closed contour $\mathcal{C}$. The expectation value in Eq. (6) is to be evaluated by the Wick rule, and the basic correlator, obtained from Eq. (2) by simple Fourier transform to configuration space, is $\left(x_{ \pm}=x_{1}\right.$ $\left.\mp i x_{2}\right)$

$$
\left\langle\left(A_{+}\right)_{i, j}(\vec{x})\left(A_{+}\right)_{k, l}\left(\overrightarrow{x^{\prime}}\right)\right\rangle=\frac{1}{4 \pi} \delta_{i, l} \delta_{j, k} \frac{x_{+}-x_{+}^{\prime}}{x_{-}-x_{-}^{\prime}} .
$$

Here we have also written out the matrix indices of the gauge field $A_{+}$.

It is simplest to consider a circular contour. Then the weighted basic correlator is independent of the variables

$$
\begin{aligned}
s, s^{\prime}: \dot{x}_{-}(s) \dot{x}_{-}\left(s^{\prime}\right)\left(x_{+}(s)\right. & \\
& \left.-x_{+}\left(s^{\prime}\right)\right) /\left(x_{-}(s)-x_{-}\left(s^{\prime}\right)\right)=(2 \pi r)^{2} .
\end{aligned}
$$

Now the integration over the path parameters $s_{1}, \ldots, s_{2 n}$ becomes trivial, and the computation can be very simply performed, say up to three loops. One finds, for $\mathrm{U}(N)$,

$$
\begin{aligned}
\mathcal{W}_{\mathcal{C}}^{\mathrm{U}(N)}= & 1-\frac{1}{2} N g^{2} \mathcal{A}_{\mathcal{C}}+\frac{1}{8}\left(\frac{2}{3} N^{2}+\frac{1}{3}\right) g^{4} \mathcal{A}_{\mathcal{C}}^{2} \\
& -\frac{1}{48}\left(\frac{5}{15} N^{3}+\frac{10}{15} N\right) g^{6} \mathcal{A}_{\mathcal{C}}^{3}+\mathcal{O}\left(g^{8}\right),
\end{aligned}
$$

where $\mathcal{A}_{\mathcal{C}}=\pi r^{2}$ is the area of the circle. Evidently this threeloop result is incompatible with the standard law Eq. (5) unless $N=1$. The technical reason is that the Abelian result is reproduced in the WML prescription by nonplanar gluon exchange; as soon as $N>1$, crossing gluon lines acquire a special weight, as is evident from Eq. (8): At $\mathcal{O}\left(g^{4}\right)$, e.g., there are two planar and one crossed diagram, whereas at $\mathcal{O}\left(g^{6}\right)$ five planar and ten crossed diagrams are present.

Is the result Eq. (8) derived for a circular contour, generally true for all simple loops of the same area? Does Eq. (8) remain valid for contours in Minkowski space, if we perform the analytic continuation $\mathcal{A}_{\mathcal{C}} \rightarrow i \widetilde{\mathcal{A}}_{\mathcal{C}}$ ? The answer to both questions is yes. The argument for the first affirmation is the invariance under area preserving diffeomorphisms [13], which should not depend on the regularization scheme. The second statement should be true since the kernels (2) and (3) 
are related by analytic continuation, and since the $\mathrm{Wu}$ kernel (2) possesses a commutativity property already emphasized in the original work [5].

Since the two above assertions are crucial for the following, we have convinced ourselves of their validity for a variety of simple contours such as ellipses, triangles, rectangles of various orientations both in Euclidean and in Minkowski space. Careful numerical evaluation of Eq. (6) up to $\mathcal{O}\left(g^{4}\right)$ and in some case up to $\mathcal{O}\left(g^{6}\right)$ yielded in all cases perfect agreement with Eq. (8) to a level of precision of $10^{-4} \cdots 10^{-3}$. Our numerical computations thus lend strong support to the formal general arguments mentioned above.

Our findings are seemingly in disagreement with the analytical two-loop [i.e., $\mathcal{O}\left(\mathrm{g}^{4}\right)$ ] calculations of Bassetto et al. [11] for rectangular contours in Minkowski space [14]. They consider two orientations of the loop. In the first case, the rectangle is oriented along the light-cone space and lightcone time axes. Agreement with Eq. (8) can be established [15]. In the second case, the rectangle is oriented along the space and time axes. Here they concluded that the WML regularization violated the area law. However, the authors failed to realize that the complicated dependence of their final result on the aspect ratio of their rectangle exactly can$c e l s$, as is easily verified. If this cancellation is taken into account, the Bassetto et al. result is in perfect agreement with ours.

We also investigated the invariance of $\mathcal{W}_{\mathcal{C}}$ under transformations to generalized axial gauges (cf. [5])

$$
\frac{1}{\left(k_{1}+i k_{2}\right)^{2}} \rightarrow \frac{1}{\left(k_{1} \cos \theta+i k_{2} \sin \theta\right)^{2}}
$$

for $0<\theta \leqslant \pi / 4$. In that case, symmetric integration amounts to cutting out a spherical hole in the $\left(\widetilde{k}_{1}, \widetilde{k}_{2}\right)$ plane with $\widetilde{k}_{1}=k_{1} \cos \theta$ and $\widetilde{k}_{2}=k_{2} \sin \theta$. The propagator becomes $x_{+} / x_{-} \rightarrow \tilde{x}_{+} / \tilde{x}_{-}$, with $\tilde{x}_{ \pm}=x_{1} / \cos \theta \mp i x_{2} / \sin \theta$. As before, the gauge invariance was checked numerically to a relative precision of about $10^{-3}$ for several values of $\theta$.

Having established that the choice of contour is arbitrary, we may restrict ourselves to the especially simple Euclidean circular contour. There we can find all further terms in Eq. (8), since the integrand in Eq. (6) is constant. The problem of determining the Wilson loop reduces to the purely combinatorial problem of finding the group-theoretic factors corresponding to the Wick contractions. Fortunately, these factors are generated by a simple matrix integral:

$$
\mathcal{W}_{\mathcal{C}}=\frac{1}{Z} \int \mathcal{D} F \exp \left(-\frac{1}{2} \operatorname{Tr} F^{2}\right) \frac{1}{N} \operatorname{Tr} \exp \left(i g \sqrt{\mathcal{A}_{\mathcal{C}}} F\right) .
$$

For $\mathrm{U}(N), \quad \mathcal{D} F$ denotes the flat integration measure on the space of Hermitian $N \times N$ matrices: $\mathcal{D} F$ $=\Pi_{i=1}^{N} d F_{i i} \Pi_{i<j}^{N} d\left(\operatorname{Re} F_{i j}\right) d\left(\operatorname{Im} F_{i j}\right) . Z$ is a normalization factor: $Z=\int \mathcal{D} F \exp \left(-\frac{1}{2} \operatorname{Tr} F^{2}\right)$. This matrix integral has been evaluated with a variety of methods [16]. The final result constitutes the exact expression for the Wilson loop at any $N$ :

$$
\mathcal{W}_{\mathcal{C}}^{\mathrm{U}(N)}=\exp \left(-\frac{1}{2} g^{2} \mathcal{A}_{\mathcal{C}}\right) \frac{1}{N} \oint \frac{d z}{2 \pi i} \exp \left(-g^{2} \mathcal{A}_{\mathcal{C}} z\right)\left(\frac{z+1}{z}\right)^{N}
$$

The contour integral, which encloses the multiple pole at $z$ $=0$, gives a Laguerre polynomial in $g^{2} \mathcal{A}_{\mathcal{C}}$ of order $N-1$ : $L_{N-1}^{1}\left(g^{2} \mathcal{A}_{\mathcal{C}}\right)$. The first few examples are

$$
\begin{gathered}
\mathcal{W}_{\mathcal{C}}^{\mathrm{U}(1)}=e^{-(1 / 2) g^{2} \mathcal{A}_{\mathcal{C}}} \\
\mathcal{W}_{\mathcal{C}}^{\mathrm{U}(2)}=\left(1-\frac{1}{2} g^{2} \mathcal{A}_{\mathcal{C}}\right) e^{-(1 / 2) g^{2} \mathcal{A}_{\mathcal{C}}} \\
\mathcal{W}_{\mathcal{C}}^{\mathrm{U}(3)}=\left(1-g^{2} \mathcal{A}_{\mathcal{C}}+\frac{1}{6}\left(g^{2} \mathcal{A}_{\mathcal{C}}\right)^{2}\right) e^{-(1 / 2) g^{2} \mathcal{A}_{\mathcal{C}}}
\end{gathered}
$$

For $\mathrm{SU}(N)$, the integration measure in Eq. (10) has to be modified to enforce tracelessness: $\mathcal{D} F \rightarrow \mathcal{D} F \delta(\operatorname{Tr} F)$. The $\mathrm{U}(1)$ part decouples from the $\mathrm{U}(N)$ Wilson loop and we find

$$
\mathcal{W}_{\mathcal{C}}^{\mathrm{SU}(N)}=\exp \left(\frac{1}{2 N} g^{2} \mathcal{A}_{\mathcal{C}}\right) \mathcal{W}_{\mathcal{C}}^{U(N)}
$$

Our result coincides with the usual expression (5) only in the Abelian U(1) case. Incidentally, this explains why $\mathrm{Wu}$ and Stamatescu [17] were able to reproduce the standard solution of the Schwinger model (i.e., two-dimensional QED) with the WML regularization.

We now study the exact formulas (11), (13) in 't Hooft's large $N$ limit: $N \rightarrow \infty, \quad g \rightarrow 0$, with fixed $\widetilde{g}^{2}=N g^{2}$. Equations (11), (13) become the integral representation of a Bessel function:

$$
\mathcal{W}_{\mathcal{C}}^{\mathrm{U}(\infty)}=\mathcal{W}_{\mathcal{C}}^{\mathrm{SU}(\infty)}=\frac{1}{\widetilde{g} \sqrt{\mathcal{A}_{\mathcal{C}}}} J_{1}\left(2 \tilde{g} \sqrt{\mathcal{A}_{\mathcal{C}}}\right)
$$

Curiously, the factors $\exp \left(-\frac{1}{2} g^{2} \mathcal{A}_{\mathcal{C}}\right)$ present at all finite $N$ [cf. Eqs. (11), (12)] disappear in the $N \rightarrow \infty$ limit. But it is precisely these factors which lead to confinement at finite $N$. Very much unlike the usual theory, where from Eq. (5) we have $\mathcal{W}_{\mathcal{C}}^{\mathrm{U}(\infty)}=\exp \left(-\frac{1}{2} \widetilde{g}^{2} \mathcal{A}_{\mathcal{C}}\right)$, in the present model the behavior changes qualitatively at $N=\infty$, as $\mathcal{A}_{\mathcal{C}} \longrightarrow \infty$ :

$$
\mathcal{W}_{\mathcal{C}}^{\mathrm{U}(\infty)} \sim \frac{1}{\sqrt{\pi}}\left(\tilde{g}^{2} \mathcal{A}_{\mathcal{C}}\right)^{-3 / 4} \cos \left(2 \tilde{g} \sqrt{\mathcal{A}_{\mathcal{C}}}-\frac{3}{4} \pi\right) .
$$

This falloff at large areas $\mathcal{A}_{\mathcal{C}}$ is far too slow to ensure confinement. We therefore conclude that Wu's model of mesons has to be studied at finite $N$; the Bethe-Salpeter equation written down in [5] is not expected to lead to a discrete meson spectrum.

What have we achieved so far? We have presented evidence that, in addition to the 't Hooft principal value prescription, the WML symmetric integration yields a consistent yet different theory. We have found this theory to be qualitatively different in the $N \rightarrow \infty$ limit. This allows us to solve 
the old riddle of why bound states for the $\mathrm{Wu}$ equation have never been found. It also allows us to expose a case in which the $N \rightarrow \infty$ limit is invalid.

At finite N, the WML prescription, while not reproducing ordinary $\mathrm{QCD}_{2}$, appears to be self-consistent, unless inconsistencies end up being found on a subtler level. It is known that $\mathrm{QCD}_{2}$ is special in that it can be considerably generalized by adding higher powers (products of traces) of the field strength to the action; such terms are irrelevant in $D>2$ butbegin to scale at exactly $D=2[12,18]$. Therefore, as already pointed out in [18], continuing a $D$-dimensional formulation of QCD down to $D=2$ might not give the usual $\operatorname{Tr} F^{2}$ theory, but one of the generalized theories $\left(G \mathrm{QCD}_{2}\right)$. However, this does not explain why there are coexisting theories with the same Lagrangian $\mathcal{L}=-\frac{1}{4} \operatorname{Tr} F^{2}$. It is easily seen that the WML prescription in Euclidean space cannot be described by a standard (even generalized) lattice gauge theory. It would be very interesting to find a lattice discreti- zation for this theory. A natural guess would be a "dielectric'" lattice gauge theory where the gauge degrees of freedom are noncompact complex matrices peaked around an appropriate measure.

We emphasize that our results raise a number of interesting further issues. First, it would be interesting to show full consistency of "Wu's alternative model for mesons,' unearthing its gauge-invariant microscopic degrees of freedom. In this case it might serve as an instructive laboratory for a bound state problem where the large $N$ approximation is invalid. Secondly, and of obvious importance, it should be understood why the straightforward limit of a prescription widely used in perturbative gauge theory in physical dimensions fails when naively continued to two dimensions.

We would like to thank I. Bars, A. Bassetto, S. Wadia and especially T. T. Wu for several inspiring discussions. W.K. thanks the CERN theory division for hospitality.
[1] G. 't Hooft, Nucl. Phys. B75, 461 (1974).

[2] C. G. Callan Jr., N. Coote, and D. J. Gross, Phys. Rev. D 13, 1649 (1976); M. B. Einhorn, ibid. 14, 3451 (1976).

[3] I. Bars and M. B. Green, Phys. Rev. D 17, 537 (1978); M. Li, L. Wilets, and M. C. Birse, J. Phys. G 13, 915 (1987).

[4] This problem does not seem to arise in the spatial axial gauge treatment of Ref. [3]. However, in order to achieve agreement with the light-cone gauge result, an unusual $i \epsilon$ prescription for the quark propagators was necessary. This again interferes with Wick rotation.

[5] T. T. Wu, Phys. Lett. 71B, 142 (1977); Phys. Rep. 49, 245 (1979).

[6] S. Mandelstam, Nucl. Phys. B213, 149 (1983).

[7] G. Leibbrandt, Phys. Rev. D 29, 1699 (1984).

[8] D. M. Capper, J. J. Dulwich, and M. J. Litvak, Nucl. Phys. B241, 463 (1984).

[9] A. Bassetto and L. Griguolo, Phys. Rev. D 53, 7385 (1996); A. Bassetto, G. Nardelli, and A. Shuvaev, Nucl. Phys. B495, 451 (1997).

[10] N. J. Bee, P. J. Stopford, and B. R. Webber, Phys. Lett. 76B, 315 (1978); R. Brout, F. Englert, and J.-M. Frère, Nucl. Phys. B134, 327 (1978); P. Mitra, Phys. Lett. 92B, 324 (1980).

[11] A. Bassetto, F. De Biasio, and L. Griguolo, Phys. Rev. Lett. 72, 3141 (1994); A. Bassetto, L. Griguolo, and G. Nardelli, Phys. Rev. D 54, 2845 (1996); A. Bassetto and G. Nardelli,
Int. J. Mod. Phys. A 12, 1075 (1997); 12, 2947(E) (1997); A. Bassetto, D. Colferai, G. Nardelli, Nucl. Phys. B501, 227 (1997).

[12] E. Witten, Commun. Math. Phys. 141, 153 (1991).

[13] This can be seen by realizing that the field strength is a twoform in two dimensions. The action is therefore invariant under all diffeomorphisms that preserve the volume element of the surface.

[14] In order to compare, one has to make the trivial change $\mathrm{U}(N) \rightarrow \mathrm{SU}(N)$ [see Eq. (13)]. An additional replacement $g^{2}$ $\rightarrow \frac{1}{2} g^{2}$ is due to a difference in normalization of the group's generators.

[15] A typographical error $(24 \rightarrow 6)$ in Eq. (8) in the third reference of [11] needs to be corrected. We thank A. Bassetto for a brief discussion concerning this point.

[16] E. Brézin, in Applications of Field Theory to Statistical Mechanics: Proceedings, Sitges, near Barcelona, Spain, 1984, edited by L. Garrido, Springer Lecture Notes in Physics Vol. 216 (Springer, Berlin, 1984), pp. 115; J. Harer and D. Zagier, Invent. Math. 85, 457 (1986); R. C. Penner, Bull. Am. Math. Soc. 15, 73 (1986); C. Itzykson and J.-B. Zuber, Commun. Math. Phys. 134, 197 (1990); V. A. Kazakov, Nucl. Phys. B354, 614 (1991).

[17] I. O. Stamatescu and T. T. Wu, Nucl. Phys. B143, 503 (1978).

[18] M. R. Douglas, K. Li, and M. Staudacher, Nucl. Phys. B420, 118 (1994). 